\title{
Stock Indices of BRIC economies: Explored for Non Linear Dynamics and Volatility
}

\author{
Mrs. Muskan Karamchandani ${ }^{1}$, Ms. Shubhra Mohadikar ${ }^{2}$, Ms. Savera Jain ${ }^{3}$ \\ 1,2,3 (Finance department-International Institute of Professional Studies, Devi Ahilya University, India)
}

\begin{abstract}
The current global scenario is a vulnerable one with crisis affecting the global markets. The speed, the magnitude and the frequency of changes has made markets more volatile than ever before. The reports of ECRI (Economic Cycle Research Institute) "We are in an era of frequent recession" ring true to ears.

Predictability in financial markets has long been rejected by the conventional theories of finance. The implicit assumption in these theories that the investors are rational and the financial markets do not possess non linear dynamics has been falsified in many researches. Lately, the tools of Econophysics have proved to successfully overcome this shortcoming. Concepts of Hurst Exponent (Rescaled range analysis) and Shannon Entropy (Symbolic Time Series Analysis) borrowed from Physics and applied in Economics have opened novel dimensions for researchers, policy makers and investors. This paper is an attempt to explore the dynamics of the major stock indices of BRIC economies with these tools. An investigation into existence of the predictability (Hurst Exponent analysis) in these emerging markets coupled with a new volatility measure (Shannon entropy) hint towards non linear dynamics. The findings provide a substantial evidence of foretelling trend reversals in Russian, Indian and Chinese markets. Also, the Indian market was found to be the least volatile amongst the 4 economies. The study would encapsulate wide implications ranging from market efficiency interpretations, to facilitating investors on deciding on their exit and entry into the market and also, to regulators on when to intervene.
\end{abstract}

Keywords: BRIC economies, Econophysics, Hurst Analysis, Shannon Entropy.

\section{Introduction}

Efficient Market Hypothesis [1] applied to financial markets had long been tested by researchers mostly to falsify the assumption. Conventional theorists regard efficiency as an unavoidable phenomenon in the financial markets. This assumes the linearity component implicit in the financial time-series. However, real world application embeds financial time-series data with both linear and non linear as well as deterministic and stochastic components. There might be instances of recurrences of patterns and so, predictability. In such a case, to improve the quality of analysis, it becomes imperative to test the time series for predictability and then apply the appropriate statistical models.

Non- linear dynamics in financial markets implies that predictability is of a non-linear nature, i.e., the effect of an event on investor's memory decay in a non linear manner. If predictability is extant, investors get more chances of earning abnormal profits by using past prices and Econophysics tools that can overcome limitations of the conventional techniques may result in availability of better forecasting tools to both investors as well as policy makers. However, one needs to justify whether predictability exists in the market or not.

Research suggests that unlike developed markets, non linear dependence has been dominant in the emerging markets [2]. This has direct consequences on the efficiency of these markets. Moreover, many researchers support the view that efficiency of markets tend to change over time. Therefore, the emerging markets need to be tested on a continuous basis to check any patterns of inefficiency/ predictability.

Since last 15 years, the BRIC economies have driven the global growth. Moreover, they are the major emerging markets in terms of GDP growth. Thus, the BRIC economies have been selected in this study to check for any latent predictability existing in these markets. The major indices of the economies' stock exchanges can provide a realistic picture of these markets. Hurst Analysis, a tool borrowed from Econophysics is best known for measuring the non linear dependence in a long term time-series data. A larger Hurst value indicates predictability in the time-series data. Also, to check this on a continuous basis, rolling window of Hurst values $(\mathrm{n}=150,215,256$ and 300) can be plotted on a graph. Further, in order to check if Hurst can also be used as a forecasting tool, it should be plotted with the closing prices of each index. If predictability could be found then the conventional theories fail to apply on these markets also making the assumption in calculating volatility unrealistic. Therefore, a new measure to calculate volatility has been introduced. Entropy, a measure of uncertainty hails from the information theory in physics but now finds prevalence in finance as well. Claude Shannon [3] was the first to postulate that entropy as a measure of uncertainty can be used in any data series where probabilities apply. His entropy measure came to be known as Shannon Entropy, an upcoming tool that can overcome the constraints regarding theoretical assumptions inherent in models widely applied by 
forecasters. Few researches provide proof of Shannon entropy being a better measure than the conventional and prevalent Standard deviation and other measures of volatility. Standard deviation and Shannon Entropy values need to be checked for these markets and the markets shall be ranked accordingly. Evidence of these can have major implications on the strategies adopted by the investors and policy makers.

The study intends to put light on evidence of predictability in the financial markets of the most dominant emerging markets as well as corroborate the same with a new measure of volatility, i.e. Shannon Entropy. Shannon Entropy might calibrate many tools (Sharpe measure, capital market line) used by investors, portfolio managers and policy makers to devise more accurate strategies.

\section{Literature Review}

Examining predictability and volatility in financial markets has been a subject of research for quite a while. At the same time, interdisciplinary research fields such as Econophysics, Financial Astronomy and Bioeconomics have also become an interesting development with an aim to solve the complex problems of the economies of today. Hurst Exponent, a tool borrowed from Econophysics is widely used as a measure of predictability in long-term financial time-series data. It was originally developed in Hydrology by H.E. Hurst in 1951, and now it finds acceptance in many areas such as applied mathematics, long memory processes and spectral analysis.

There have been a number of studies that uses Hurst Exponent to measure predictability in financial markets. A significant one was undertaken by [4]. Another study was made by [5] in which Hurst was calculated by using Rescaled range analysis and predictability was investigated for NASDAQ. Hurst Exponent can also be used as a complementary with other tools. In our previous paper [6] Hurst was used in synthesis with technical analysis to generate profit signals for foreign exchange market.

The above mentioned studies used Hurst Exponent analysis as a static measure. However, [7] who investigated Hurst in foreign exchange market for currencies like British Pound, Canadian Dollar, etc found that majority of foreign currencies exhibit Hurst exponent that is statistically different from 0.5 and the value changes over time. [8] used this time varying Hurst analysis to detect long term correlation in financial time series. The technique was applied in a rolling window on Dow Jones index and it was argued that Hurst may be used to detect crises. Long range dependence for eleven emerging countries and for US and Japan was tested for period of 1992 to 2002 by [9]. They used rolling sample approach to analyze dynamics of Hurst Exponent over time and results suggested that Asian equity markets show greater inefficiency than that of Latin America. Other finding was that only India had shown the upward Hurst trend in the time period. Furthermore, [10] implemented Hurst Exponent analysis for investigating efficiency of Brazilian foreign exchange market and [11] used the method to analyze dynamical behaviour of US stock markets Dow Jones.

[2] used time and scale Hurst exponent analysis on the financial time series of worldwide markets and found that mature markets have Hurst values around 0.5 and emergent markets have Hurst values well above 0.5. Thus, a distinction was established between emerging markets and mature markets showing that mature markets are more efficient than emergent markets. Also, [12] used Hurst with nearest neighbour prediction method to calculate hit rate for prediction of direction of future prices of 60 market indices of various countries.

However, only by knowing the market's predictability will not fulfil the purpose of the study. One also needs to know the volatility of these markets and that too with an effective tool. Traditional method of standard deviation is based on certain constraints that financial time-series do not show non-linear dependence. However, in real world applications, this might not be true. [13] inferred that capturing the intrinsic non linear effects in the financial time-series may improve forecasting. Therefore, in order to get accuracy from standard deviation, we need to check it for non-linear dynamics.

Econophysics provide certain tools which do not contain such constraints, one of them being Shannon entropy. The investigation of financial time series for long memory and volatility clustering using Shannon entropy was done by [14]. They used the non linear models Arch, Garch and Figarch to check volatility in the US and European markets and then they applied entropy models to corroborate the results from the above stated models. They concluded that entropy measures could provide a clearer idea of the uncertainty in financial time series because they do not impose any constraints on the theoretical probability distribution, whereas, the Arch/ Garch models are based on the assumption that all variables are independent and identically distributed.

[15] further explored the concept and used the combination of Hurst and Shannon entropy in order to detect major trend reversal points in the three emerging markets within Nasdaq OMX Baltic stock exchange. Furthermore, they used the concept of neural networks and symbolized time series analysis to calculate modified Shannon Entropy. However, the inefficiency and volatility in the BRIC economies still remains unexplored and thus, are considered to be the subject of this study. 


\section{Objectives}

The study intends to analyze predictive nature of financial time series of stock indices of BRIC economies by use of Hurst Exponent analysis and level of correlation among returns of these economies for the period 1 January, 2006 to 31 August, 2013. Moreover, volatility is also calculated for the same by a tool borrowed from information theory, i.e. Shannon Entropy. Thus, the objectives can be stated as:

- To study Non Linear Dynamics of BRIC economies by Hurst Exponent analysis by the use of Static and Rolling Hurst values.

- To study volatility in BRIC economies by Shannon Entropy analysis.

\section{Methodology}

Major indices of the stock exchanges of the BRIC economies (Ibovespa, Micex, Sensex and Shanghai index respectively) are tested separately by the tools used in the study.

\subsection{Return calculation and Descriptive statistics of returns 4.1.1 Return calculation}

The use of raw daily price data in the stock market has many problems as movements are generally non-stationary [16], which interferes with the estimation of the Hurst Exponent. The market index series are transformed into rates of return to overcome these problems. This will provide the best view of fluctuations and volatility in the markets. Therefore, return series for all the stock indices has been generated, by using equation 1. The closing price series of Ibovespa, Micex, Sensex and Shanghai were taken since January 2006 to August 2013.

Where,

$$
\text { Return, } \mathrm{R}_{\mathrm{t}}=\operatorname{Ln}\left\{\frac{\mathrm{Yt}+1}{\mathrm{Yt}}\right\} \times 100
$$

$\mathrm{R}_{\mathrm{t}}$ is return for the current day

$\mathrm{Ln}=$ Natural logarithm

$\mathrm{Y}_{\mathrm{t}+1}$ is current day's closing price, and

$\mathrm{Y}_{\mathrm{t}}$ is previous day's closing price.

In order to have a broader understanding of the markets, descriptive statistics were calculated for the four indices studied.

\subsection{Non Linear Dynamics by Hurst Exponent Analysis}

Studies involving the Hurst Exponent were originally developed in hydrology for the practical matter of determining optimum dam sizing for the Nile River's volatile rain and drought condition that had been observed over a long period of time. The name "Hurst Exponent," or "Hurst Coefficient," derives from Harold Edwin Hurst (1880-1978), who was the lead researcher in these studies and also made the use of the standard notation $H$ for the coefficient.

The Hurst Exponent occurs in various areas of applied mathematics, including fractals and chaos theory, long memory processes and spectral analysis. Hurst Exponent estimation has also been applied in areas ranging from biophysics to computer networking.

Presence or absence of predictability in BRIC stock markets could have strong implications for market efficiency. Hence, predictability is analyzed by calculating Hurst Exponent using Rescaled Range Analysis.

\subsubsection{Hurst Exponent calculation with Rescaled Range analysis (for entire series)}

The basis of the Rescaled range analysis was laid by [17] and was further examined and elaborated by [18], [19], [20] and [21]. The Rescaled range is a statistical measure of the variability of a time series introduced by the British hydrologist Harold Edwin Hurst (1880-1978). Its purpose is to provide an assessment of how the apparent variability of a series changes with the length of the time-period being considered.

The $H$ measures dependencies in time series and is calculated through rescaled range analysis $(\mathrm{R} / \mathrm{S}$ analysis) in the study.

To estimate the Hurst Exponent, first an estimate of the dependence of the rescaled range on the time span $n$ observations is calculated. A time series of full length $N$ is divided into a number of shorter time series of length $n=N, N / 2, N / 4, \ldots$ The average rescaled range is then calculated for each value of $n$.

For a (partial) time series of length $\mathrm{n}, \mathrm{X}=X_{1}, X_{2}, \ldots, X_{n}, \mathrm{R} / \mathrm{S}$ analysis can be calculated by following steps. Firstly, the mean value $m$ is calculated: 
$\mathrm{m}=\frac{1}{n} \cdot \sum_{i=1}^{n} X_{i}$

where,

$X_{i}$ is the daily return series of Index under study

After this, a mean adjusted return series $\mathrm{Y}_{\mathrm{t}}$ is generated:

$\mathrm{Y}_{\mathrm{t}}=\mathrm{X}_{\mathrm{t}}-\mathrm{m}$,

Where,

$\mathrm{t}=1,2, \ldots, \mathrm{n}$

Then, the cumulative deviation series $\mathrm{Z}$ is calculated:

Where,

$$
Z_{t}=\sum_{i=1}^{t} Y_{i}
$$

$t=1,2, \ldots, n$

Further, the range series $\mathrm{R}$ is calculated:

$R_{t}=\max \left(Z_{1}, Z_{2}, \ldots, Z_{t}\right)-\min \left(Z_{1}, Z_{2}, \ldots, Z_{t}\right)$,

$t=1,2, \ldots, n$

Next, the standard deviation series $\mathrm{S}$ is calculated:

$\left.\mathrm{S}_{(\mathrm{t})}=\sqrt{\frac{1}{t} \sum_{i=1}^{t}\left(X_{i}\right.}-m\right)^{2}$

$t=1,2, \ldots, n$

Finally, the rescaled range series (R/S) can be estimated:

$(\mathrm{R} / \mathrm{S})_{\mathrm{t}}=\mathrm{R}_{\mathrm{t}} / \mathrm{S}_{\mathrm{t}}$

$t=1,2, \ldots, n$

And it is averaged over all the partial time series of length $n$ to get $E(R / S)$.

Hurst found that R/S actually scales by power law and he formulated the following relationship:

$\mathrm{E}(\mathrm{R} / \mathrm{S})=\mathrm{C}^{*} \mathrm{n}^{\mathrm{H}}$

As $n \rightarrow \infty$.

Where,

$\mathrm{R} / \mathrm{S}=$ Rescaled Range,

$\mathrm{C}=$ Constant, and

$\mathrm{H}=$ Hurst Exponent

Further the Hurst is calculated with the following regression equation [22]:

$\log (\mathrm{R} / \mathrm{S})_{\mathrm{n}}=\log \mathrm{C}+\mathrm{H} \log \mathrm{n}$

Here, $\log (\mathrm{R} / \mathrm{S})_{n}$ is regressed with $\log \mathrm{n}$ using linear least squares method and the slope so obtained is the Hurst Exponent.The values of the Hurst Exponent range between 0 and 1.

A Hurst Exponent value $\mathrm{H}$ close to 0.5 indicates a random walk (a Brownian time series). In a random walk there is no correlation between any element and a future element and there is a $50 \%$ probability that future return values will go either up or down. The series of this type are hard to predict.

A Hurst Exponent value $\mathrm{H}$ between 0 and 0.5 exists for time series with "anti-persistent behavior". This means that an increase will tend to be followed by a decrease (or a decrease will be followed by an increase). This behavior is sometimes called "mean reversion" which means future values will have a tendency to return to a longer term mean value. The strength of this mean reversion increases as $\mathrm{H}$ approaches 0 .

A Hurst Exponent value $\mathrm{H}$ between 0.5 and 1 indicates "persistent behavior", that is the time series is trending. If there is an increase from time step [t-1] to [t] there will probably be an increase from [t] to $[\mathrm{t}+1]$. 
The same is true of decreases, where a decrease will tend to follow a decrease. The larger the $\mathrm{H}$ value, the stronger the trend. Series of this type are easier to predict than series falling in the other two categories.

Mature markets often have Hurst Exponents closer to 0.5 than emerging markets indicating that they are more efficient and less predictable. The two approaches suggested by [9] are utilized and the Hurst Exponent is calculated:

(a) Statically (as the usual approach) and also

(b) Dynamically (rolling sample approach).

Therefore, for each of the index studied, two different results will be presented. Thus, the 4 return series of the BRIC stock market indices studied would give 4 static Hurst Exponents and another 4 Rolling Hurst Exponent series.

(a) Hurst Exponent analysis with static approach

The Rescaled range analysis [Equations 2 -9] is utilized for calculating Hurst Exponent. There is just one Hurst Exponent value calculated for each of the series under study in the static approach. In this way for the 4 indices, the 4 return series generated 4 Hurst Exponent values. The return series dated from $1^{\text {st }}$ January 2006 to $30^{\text {th }}$ August 2013.

The value of Hurst Exponent has direct implications for market efficiency and hence the $\mathrm{H}$ values are of interest to academicians as well as market participants. Actually, if the stock returns present predictability, the efficient market hypothesis is not valid anymore. Since, stock markets are dynamic in nature, the findings of [9] and [23] seems to be acceptable that market efficiency (predictability) seems to evolve over time.

(b) Hurst Exponent analysis with dynamic approach (Rolling Hurst Exponent)

This approach serves the purpose of investigating if the non linear dynamics changes over time and hence, is market efficiency a time varying concept? Earlier researches supported the idea of all or none market efficiency. With this rolling sample approach, a series of Rolling Hurst Exponent is generated. This series is plotted to infer graphically if (1) the market has shown majorly persistent or anti-persistent behaviour from the period January 2006 to August 2013, and (2) there can be suitable signals generated for foretelling index price movements by plotting it with the closing prices of each index.

In the rolling sample approach, four rolling windows of 150, 215, 256 and 300 observations were created. These were the size limits for calculation of Hurst values during the period for each of the indices. For instance, in the rolling window of 256 observations, in the first value of Hurst Exponent, calculation was based on observation number $1^{\text {st }}$ to $256^{\text {th }}$. In the calculation of second Hurst Exponent value, the $1^{\text {st }}$ observation was omitted and observations from $2^{\text {nd }}$ to $257^{\text {th }}$ were taken. This rolling window approach was followed till the last observation available was utilized. The rescaled range approach, as discussed earlier, was used for rolling Hurst value calculations.

\subsubsection{Graphical interpretation of plots of closing index values and Rolling Hurst Exponent values}

The rolling sample approach, described earlier created a Rolling Hurst Exponent series for the indices under study. For instance, a rolling window of 256 observations gave a Hurst Exponent value for every 256 observations. This rolling window approach was followed till the last observation available was utilized. The Rescaled range approach, as discussed earlier, was used for rolling Hurst value calculations.

An attempt was made to investigate if the rolling Hurst parameters could be used for prediction of index price movements by plotting of Rolling Hurst Exponent series of every index with closing price series of the corresponding Index for the period 2006-2013.The period length (2006-2013) was so chosen to have recent most plots and recover dynamics /patterns for recent years.

The motive behind these 4 simultaneous plots (of Rolling Hurst Exponent series and closing index prices) was two-fold.

- Firstly, to graphically and visually study if the Hurst Exponent could be a decisive predictor of Stock index trend reversals.

- Secondly, to visualize with graphs if the Rolling Hurst Exponent is time dependent variable. If yes, it can be inferred that even the market efficiency is continuously evolving for the stock markets.

\subsection{Correlation coefficient}

Karl Pearson's coefficient of correlation is considered to find correlation among all four indices' return series. The purpose to calculate correlation is to check whether these markets' return series show co-movements with each other. It is calculated by the following equation: 
$C_{x y}=\frac{\sum_{i=1}^{n}\left(x_{i}-<x>\right)\left(y_{i}-<y>\right)}{(n-1) S_{x} S_{y}}=\frac{\sum_{i=1}^{n}\left(x_{i}-<x>\right)\left(y_{i}-<y>\right)}{\sqrt{\sum_{i=1}^{n}\left(x_{i}-<x>\right)^{2} \sum_{i=1}^{n}\left(y_{i}-<y>\right)^{2}}}$

Here, $\langle\mathrm{x}\rangle$ and $\langle\mathrm{y}\rangle$ are the mean values of $X$ and $Y, s_{x}$ and $s_{y}$ are the standard deviations of $X$ and $Y$.

\subsection{Volatility by Shannon Entropy analysis}

Entropy is a measure of information content. Named in honour of Claude E. Shannon (known as "the father of information theory"), Shannon Entropy is a measure of informational efficiency. The measure was originally proposed by Claude E. Shannon (1948) in paper "A Mathematical Theory of Communication". The measure quantified the entropy (information content) in strings of text. The idea was that the more different letters there are, and the more equal their proportional abundances in the string of interest, the more difficult it is to correctly predict which letter will be the next one in the string. The Shannon entropy quantifies this uncertainty (entropy or degree of surprise) associated with this prediction.

The idea behind Symbolic Time Series Analysis is simple: the values of a given time series data are transformed into a finite set of symbols obtaining a finite string. Then, tools from information theory and symbolic dynamics can be used to process the symbolic sequence. In present days symbolic dynamics is used in a variety of fields including ergodic theory, topological dynamics, complex dynamics, information theory and coding. The approach, introduced in [24], [25], [26] and [27] has several successful applications to complex time series data in different areas like physics, astronomy and engineering as well. Building on this previous work, known methods have been adapted to economic time series data.

In order to recognize time patterns in complex dynamical processes a language is needed, in which to express these patterns and this is done using data symbolization. Once the data symbolization is accomplished the resulting symbolic sequence, is analysed looking for regularities to describe the dynamical behaviour of the system. The main property of data symbolization is the fact that no assumptions about the structure of the underlying dynamical system must be stated; that is, the method applies for deterministic or stochastic, linear or non-linear systems.

An evolving strand of research has found wide application of concepts of physics/information theory in finance. The major advantage of Entropy that it is not dependent upon any particular distribution, i.e., it is distribution free and thus, avoids the introduction of errors through the fitting of the distribution.

The first step for Shannon Entropy calculation involves conversion of return series into symbols. Symbolic Time Series Analysis (STSA) is then applied to recover the dynamics of the process.

The returns are symbolized so as to remove the noisiness of the data. A binary transformation is sought, and values are symbolized either 1 or 0 . A thresh hold is decided and depending on whether the value falls above or below the thresh hold, 0 or $1 \mathrm{symbol}$ is allotted. In case of financial return series the average return of the return time series under study is decided as the thresh hold. Return values above it are denoted as 1 and rest as 0 . In this way, transformed symbolic time series were created for the 4 return series of indices. Shannon Entropy (SE) is then calculated by given equation [28]:

$\mathrm{SE}=-\left[p \log _{2} p+(1-p) \log _{2}(1-p)\right]$

Where,

$p$ is the probability of returns greater than the thresh hold,

$(1-p)$ is the probability of having equal or lesser returns than the thresh hold,

and, Logarithm to the base of 2 is taken as the Symbolic Time series transformation done is binary.

The base of the logarithm used when calculating the Shannon entropy can be chosen freely. Shannon himself discussed logarithm bases 2, 10 and $e$, and these have since become the most popular bases in applications that use the Shannon entropy. Each log base corresponds to a different measurement unit, which have been called binary digits (bits), decimal digits (decits) and natural digits (nats) for the bases 2, 10 and $e$, respectively.

As the equation is concave, the maximum is obtained for $p=1 / 2$ (maximum uncertainty/volatility) and the minimum is shown when one of the events is a certain. The entropy values lie between 0 and 1 . When the entropy value is close to 1 , it means the process is random as no event is more frequent than another.

\section{Data Set and Analysis}

Non linear dynamics and volatility are studied for the stock exchanges of BRIC economies, the major market indices being Ibovespa, Micex, Sensex and Shanghai respectively. Thus, a total of 4 financial time series were taken for the study, with each index contributing a single time-series. Closing prices data of these indices are collected from Google finance for the period from $1^{\text {st }}$ January 2006 to $31^{\text {st }}$ August 2013. The period undertaken for study has been a period of turmoil for the world. This is in line with two global crises, the Subprime crisis and the Eurozone crisis. 
6.1. Descriptive Statistics

\section{Findings and Discussion}

\begin{tabular}{|c|c|c|c|c|c|c|c|}
\hline \multicolumn{8}{|c|}{ Table 1 : Descriptive Statistics } \\
\hline & $\mathrm{N}$ & Minimum & Maximum & Mean & $\begin{array}{c}\text { Standard } \\
\text { Deviation }\end{array}$ & Skewness & Kurtosis \\
\hline Sensex & 1890 & -11.60 & 15.99 & .04 & 1.71 & .129 & 7.11 \\
\hline Ibovespa & 1895 & -12.09 & 13.68 & .02 & 1.88 & -.025 & 6.17 \\
\hline Micex & 1761 & -20.66 & 25.23 & .02 & 2.42 & -.109 & 16.30 \\
\hline Shanghai & 1909 & -9.26 & 9.03 & .03 & 1.76 & -.405 & 3.35 \\
\hline
\end{tabular}

Fig.1: Plot of Risk-Return of the BRIC's stock indices.

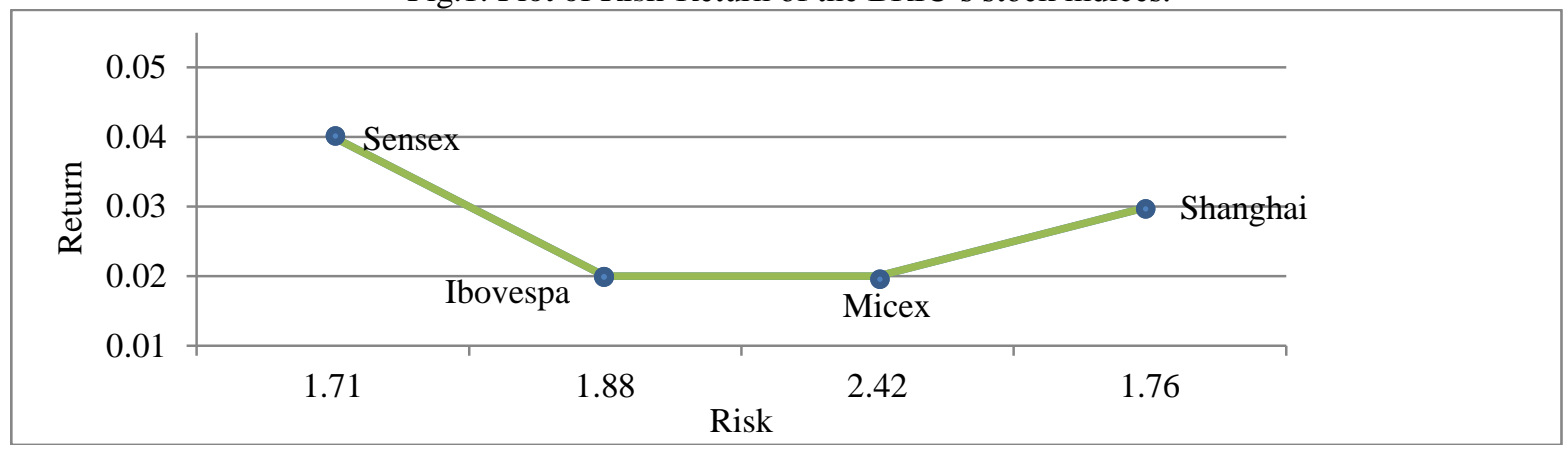

From Table 1, one can note that in terms of risk, Russian market index Micex tops amongst the countries, but when it comes to mean return, Indian market index, Sensex has been able to deliver the highest for the given period. Also, as depicted in figure 1, Sensex is the most efficient index amongst the 4 indices studied as it is able to offer the highest return with minimum risk. Another observation is that investing in Ibovespa is less risky than Micex while both provides the same average daily return. Further, from skewness statistic, one can derive that for the Sensex market, most returns are concentrated to the left of the mean, i.e. positively skewed, whereas other markets show negative skewness. Other notable thing is that except Shanghai index, all other indices show leptokurtic distribution, i.e. there is high probability of extreme values for these markets, as most of the values are concentrated around the mean.

\subsection{Hurst Exponent Analysis}

6.2.1 Hurst Exponent analysis with static approach

\begin{tabular}{|c|c|c|}
\hline \multicolumn{3}{|c|}{ Table 2 : Hurst Exponent values with static approach } \\
\hline Index & Hurst Values & Hurst Rank \\
\hline Ibovespa & 0.54 & 2 \\
\hline Micex & 0.55 & 3 \\
\hline Sensex & 0.56 & 4 \\
\hline Shanghai & 0.64 & 2 \\
\hline
\end{tabular}

Table 2 illustrates the static Hurst values for the four indices under study. According to the above Hurst estimates obtained, in terms of efficiency, Ibovespa is the most efficient market, whereas Shanghai is the least. This could be attributed to the younger age of the Russian and Chinese stock markets [30]. The Russian and Chinese stock markets were opened in 1995 and 1990 respectively. Whereas, Brazilian and Indian stock markets were founded in 1895 and 1875 respectively.

6.2.2 Hurst Exponent analysis with dynamic approach

\begin{tabular}{|c|c|}
\hline \multicolumn{2}{|c|}{ Table 3: Rolling sample Hurst values } \\
\hline Index & Percentage of Hurst values above 0.5 \\
\hline Ibovespa & 87.09 \\
\hline Micex & 91.81 \\
\hline Sensex & 93.02 \\
\hline Shanghai & 98.63 \\
\hline
\end{tabular}

Hurst values for the rolling window of 300 were calculated and plotted on the graph. Also, the percentage for values above 0.5 was calculated for each index. 
Fig. 2: Hurst values for Brazil Stock Exchange

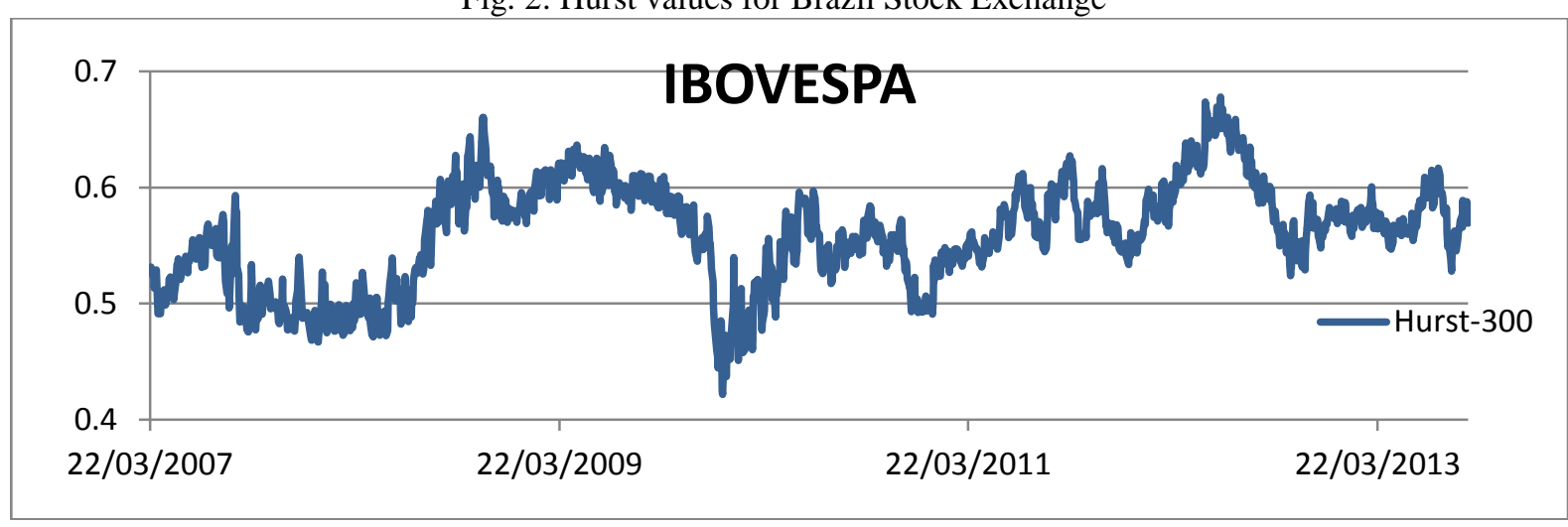

Fig. 3: Hurst values for Russian Stock Exchange

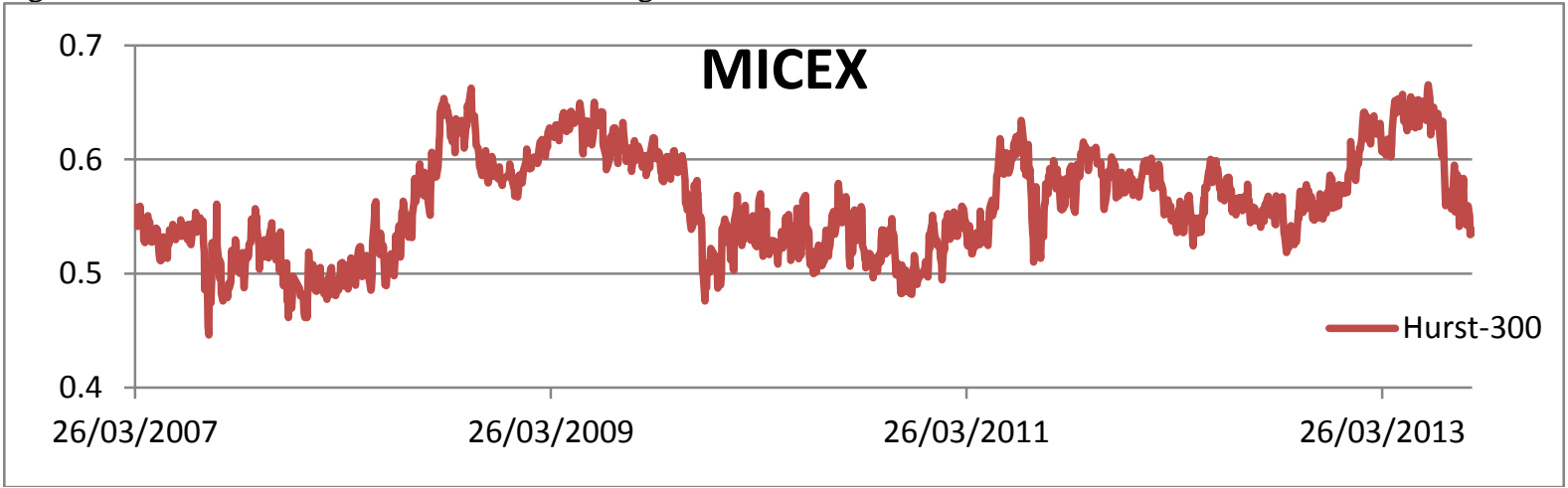

Fig. 4: Hurst values for Indian Stock Exchange

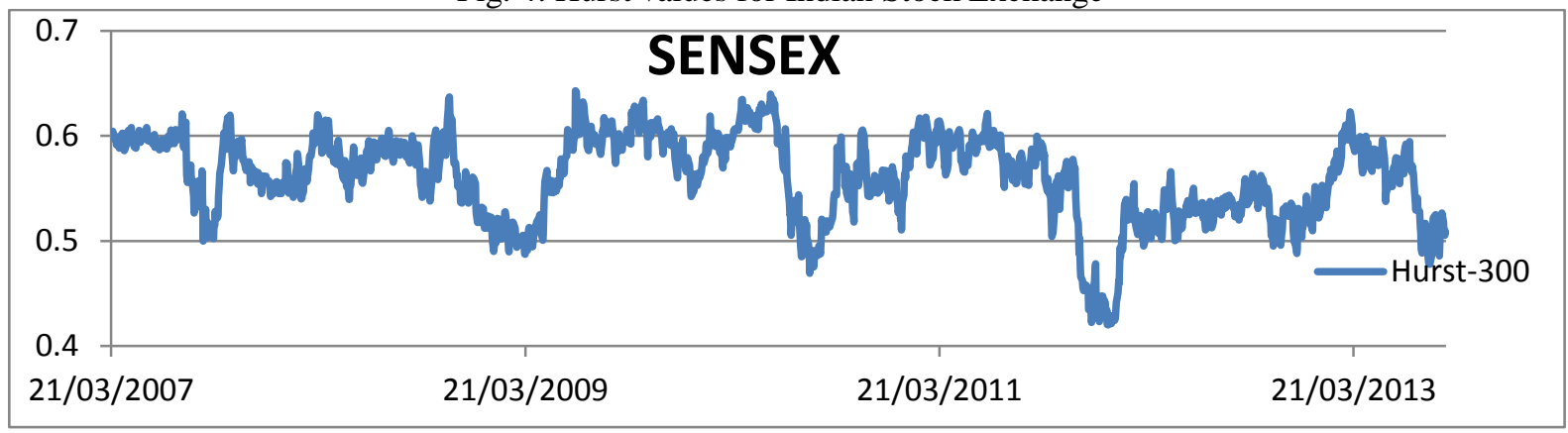

Fig. 5: Hurst values for China's Stock Exchange

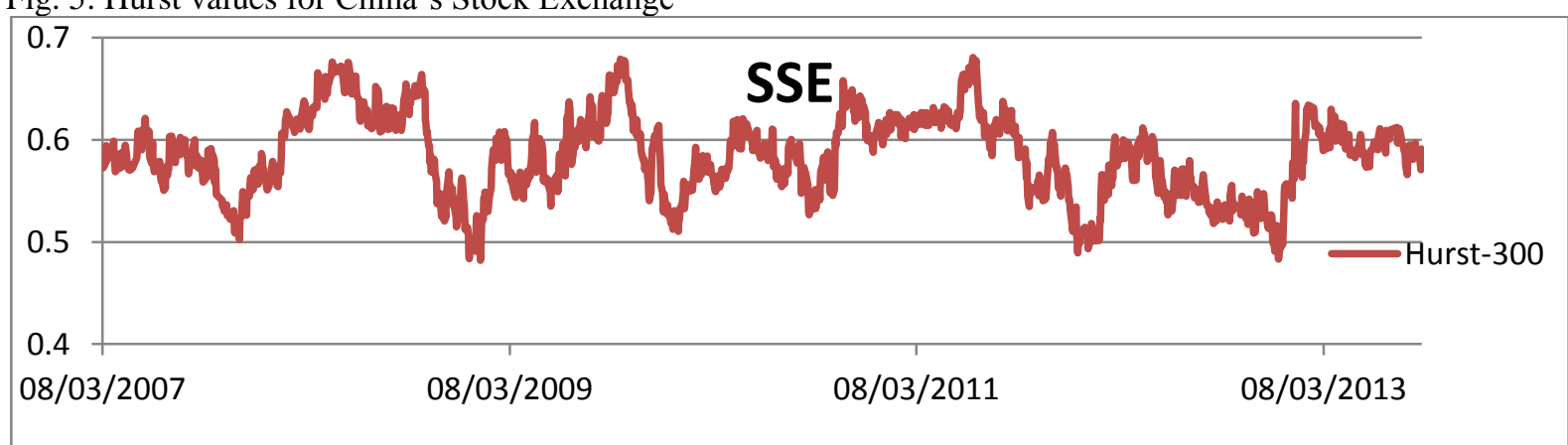

From Table 3 and the above figures, it seems that China's index Shanghai is more predictable as $98 \%$ of values were above 0.5 indicating that maximum predictability amongst BRIC stock markets exist in this market. 6.2.3 Rolling Samples Approach tested for forecasting movements 
Fig. 6: Graphs showing rolling Hurst values along with closing prices of BRIC indices for different window sizes:

Fig. 6 (a): BSE closing prices along with Hurst values for rolling window of size 300

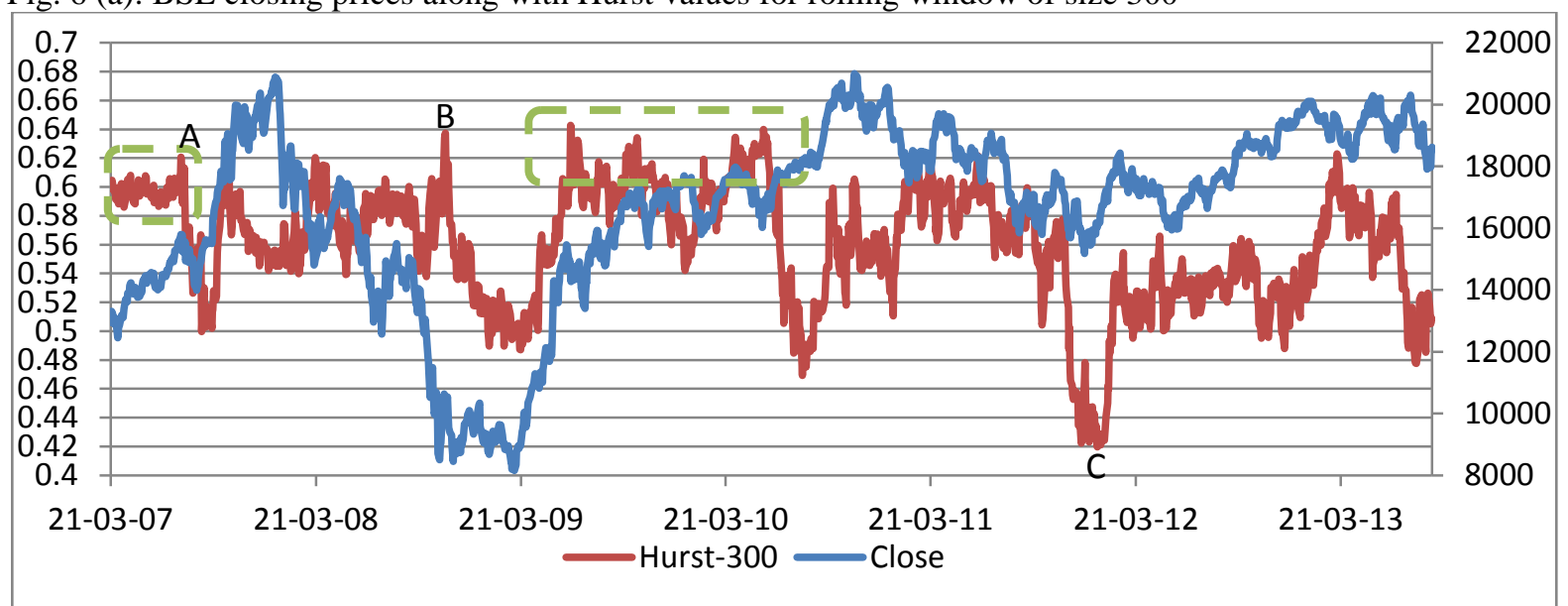

Fig. 6(b): IBOVESPA closing prices along with Hurst values for rolling window of size 150

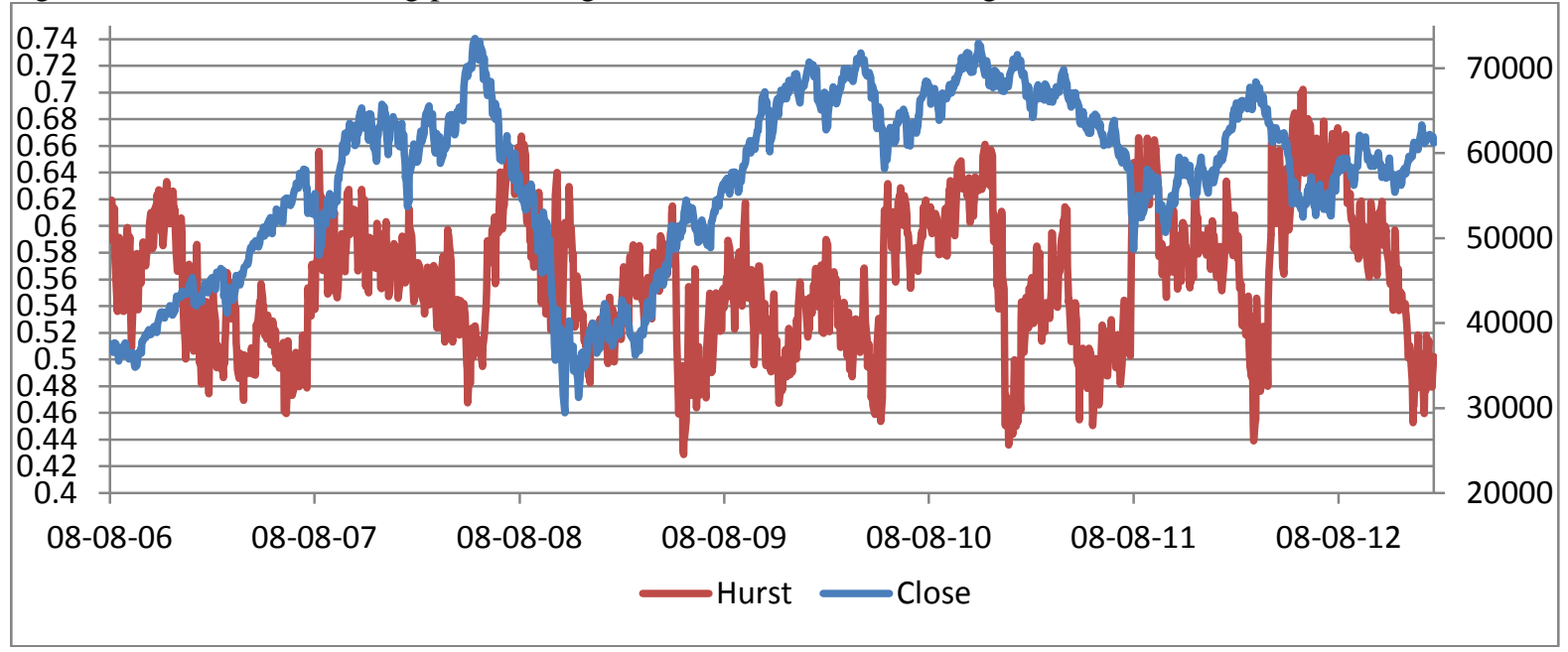

Fig. 6(c): MICEX closing prices along with Hurst values for rolling window of size 300

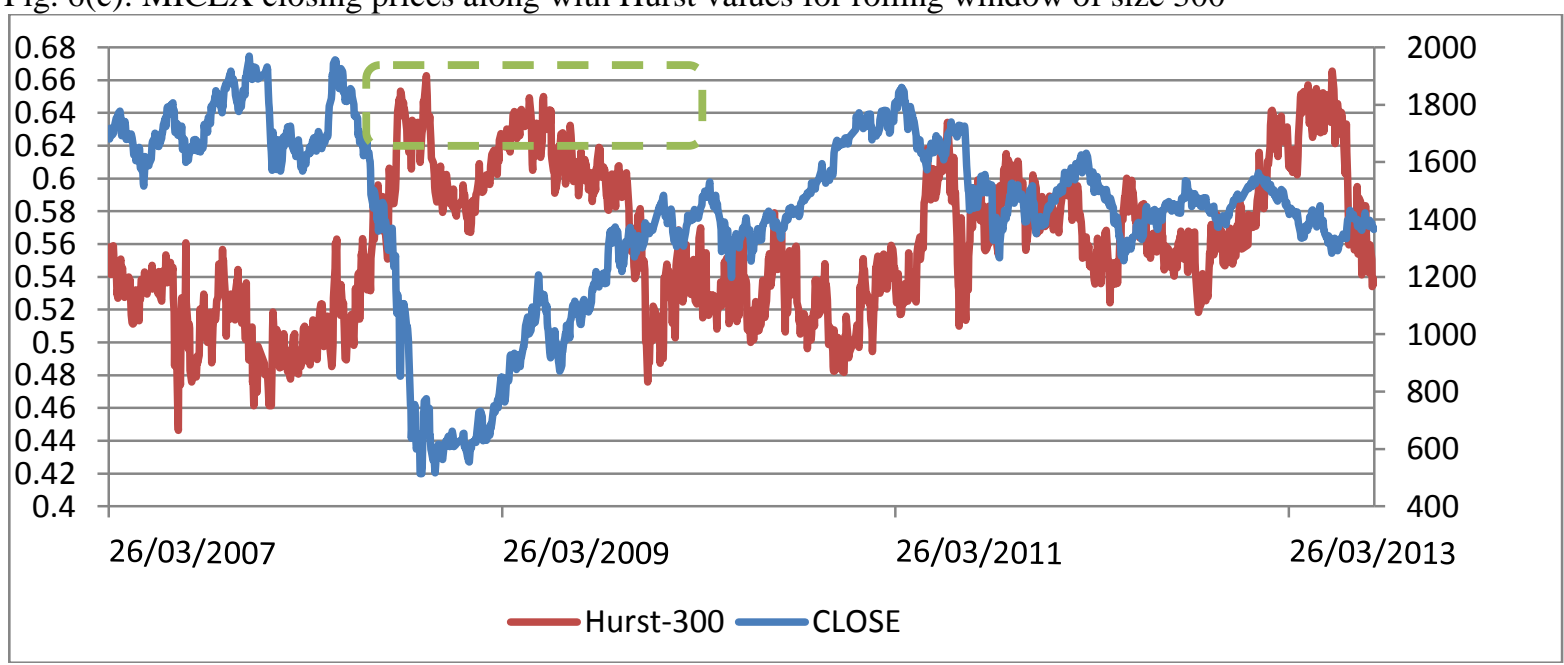


Fig. 6(d): MICEX closing prices along with Hurst values for rolling window of size 150

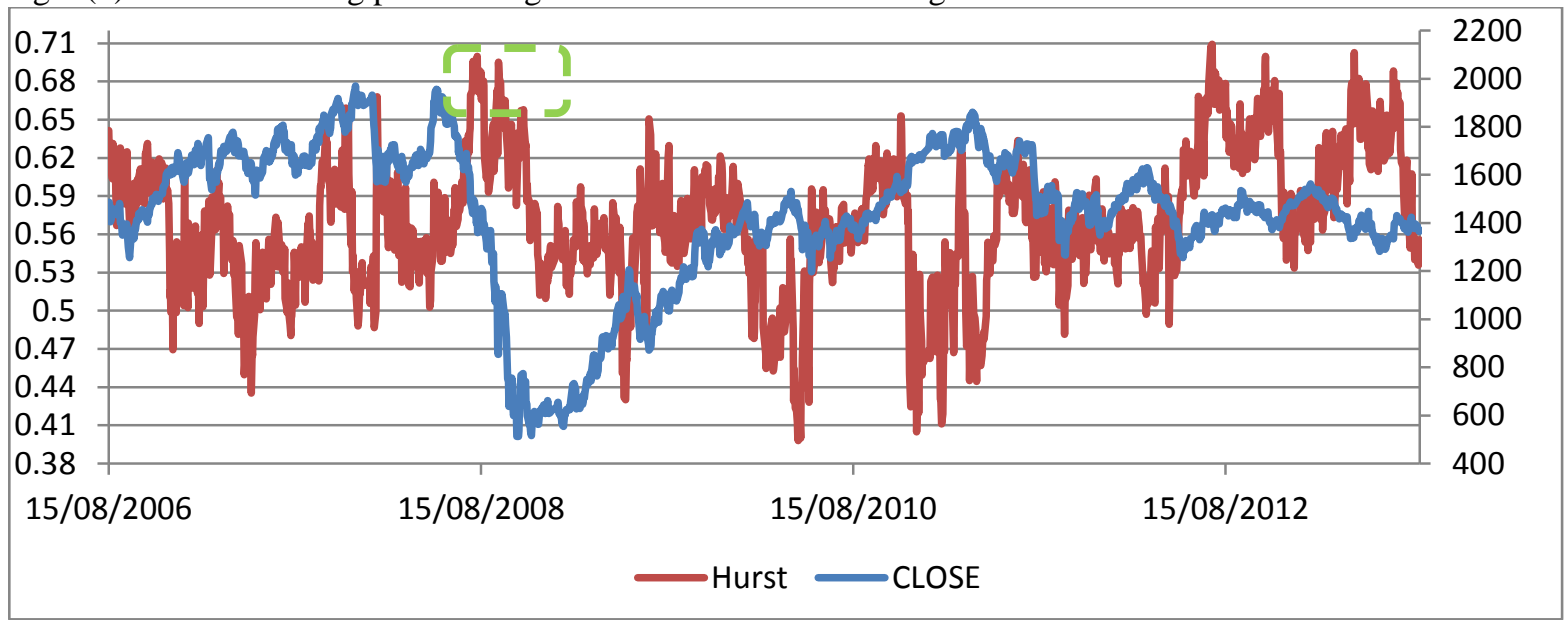

Fig. 6(e): Shanghai closing prices along with Hurst values for rolling window of size 300

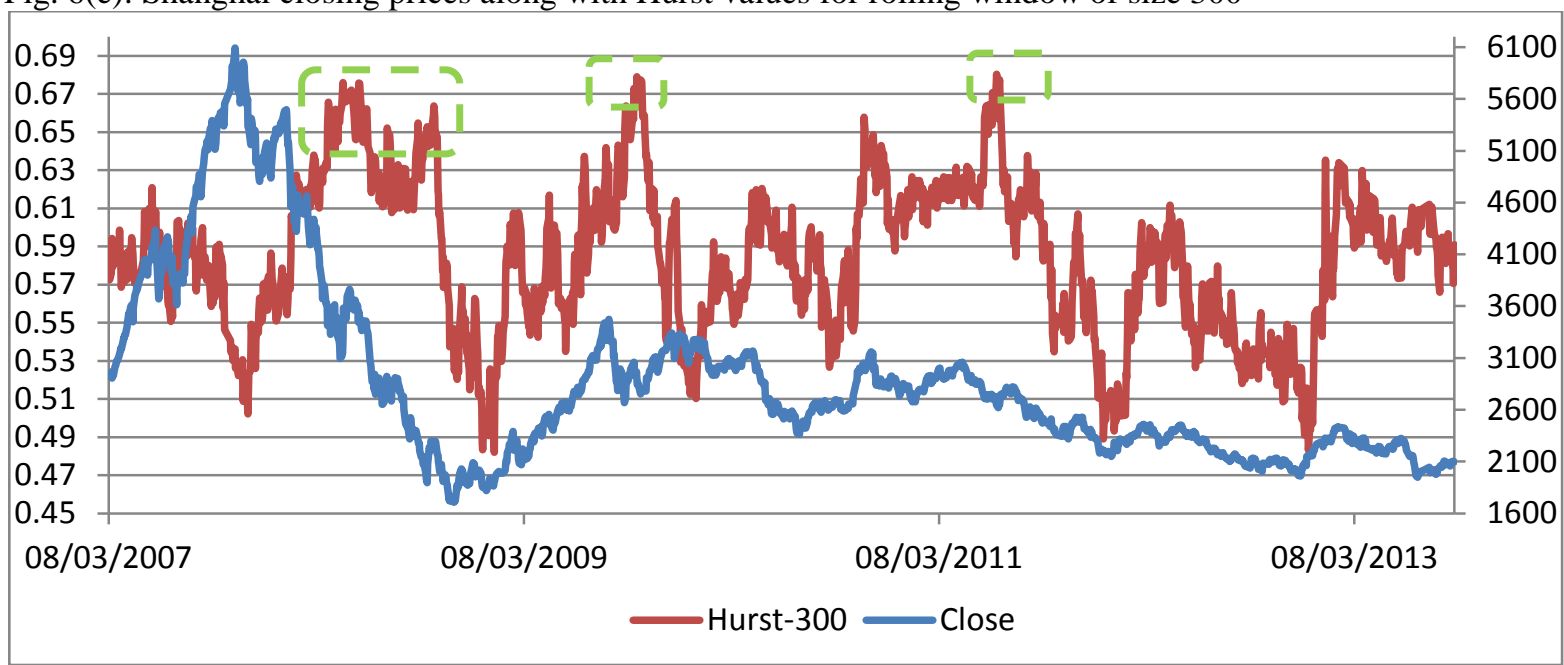

Fig. 6(f): Shanghai closing prices along with Hurst values for rolling window of size 256

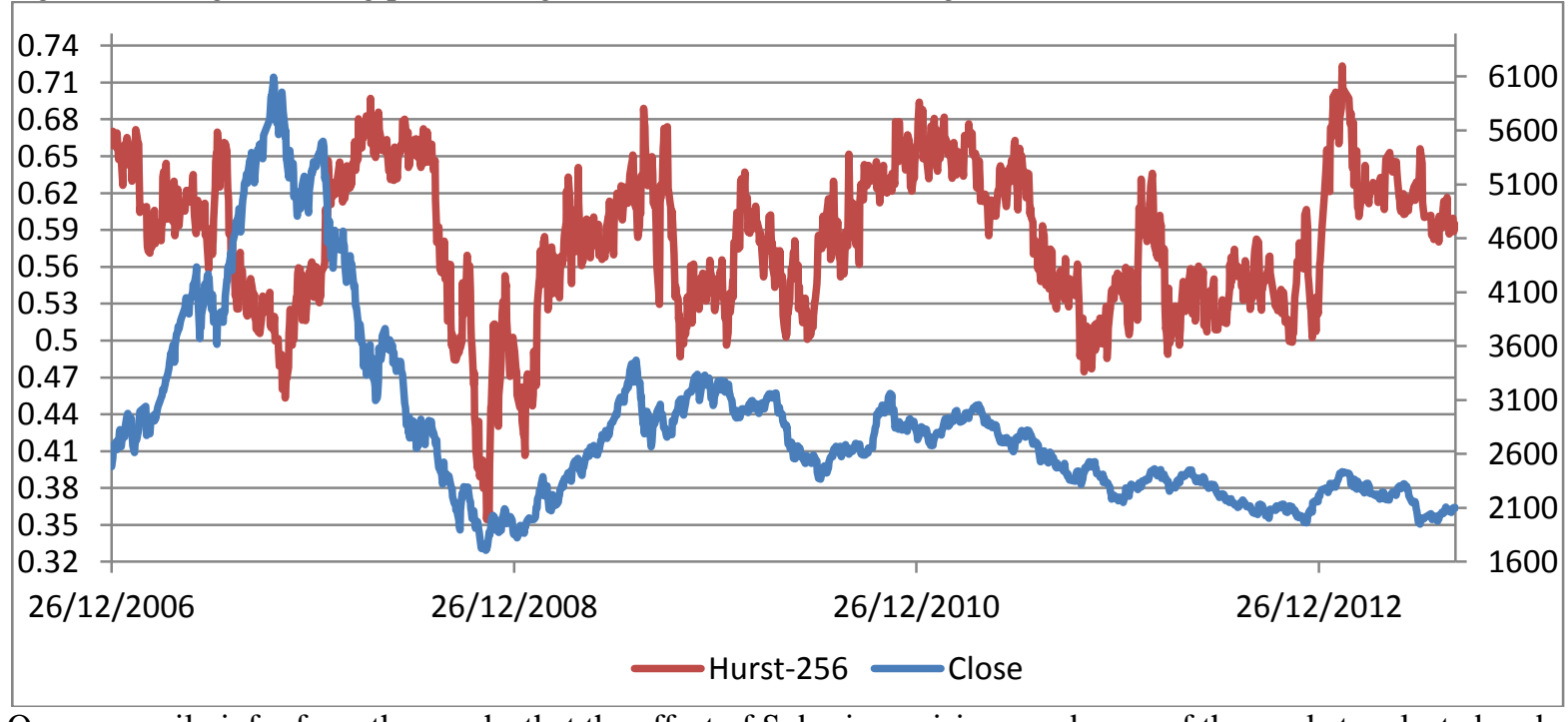

One can easily infer from the graphs that the effect of Subprime crisis spared none of the markets selected under the study. Interestingly, in all the markets, Hurst values were well above 0.5, when the stock prices fell to record lows, indicating persistence behaviour during the crisis. Although Hurst values could not predict minor 
corrections, or foretell about the timing of trend reversal, it did help in getting vital hints or signals for investors and policy makers about where the market is going and how it will go on.

From figure 6(a), one can observe the Sensex closing prices went up from March 2007 to December 2007, and the Hurst values also remained about $19.46 \%$ above 0.5 , indicating long memory. However, from point A, the Hurst value abruptly fell to 0.5 indicating restoration of efficiency in the market, and then, it again climbed up and came down giving a strong hint of trend reversal, i.e., the uptrend will not continue for a long time. From late 2007, the downfall of closing prices began and were followed by minor corrections, but for the period being, the Hurst values stubbornly remained above 0.5 (average 16.25\%), predicting continuance of the downtrend. Later on, from point B, the Hurst values again fell back to 0.5 and then again climbed up, indicating that the period of downtrend has stalled and now the uptrend will further continue. Similar pattern could be observed in the later period, although the trend was little different. However, at point $\mathrm{C}$, where Hurst fell to about 0.4 , indicated a signal of anti- persistency in the market, and then Hurst values mostly concentrated around 0.5 .

In figure 6(b), the Ibovespa index showed little coordination with the Hurst values. For either of the window sizes, there were no significant similar patterns observed. For window size of 150 , some patterns were observed. From August 2006 to August 2008, the Hurst values were on an average $8 \%$ above 0.5, indicating long memory. However, no concrete patterns were able to be generated from the same.

Figure 6(c) and 6(d) shows graphs for comparing Hurst values and Moscow Index MICEX. From figure 6(c), one can observe long memory in the market from Hurst values being constantly well above 0.5 for the period of crisis. Later on, the Hurst values again were concentrated around 0.5 indicating efficiency in the market. By having a closer look at figure 6(d), similar result was found. The green block shows that as the prices fell consistently, the Hurst values came well above 0.5.

Figure 6(e) and 6(f) showed the best results. For the rolling window size of 300, one can clearly observe the patterns as per Hurst values. The green block indicates persistence in the closing Shanghai index prices. Not only this, there was signal seen for long range trend reversal. For the rolling window size of 256, similar results were obtained.

\subsection{Correlation coefficient}

\begin{tabular}{|c|c|c|c|c|}
\hline \multicolumn{5}{|c|}{ Table 4: Correlation } \\
\hline Index Name & Ibovespa & Micex & Sensex & Shanghai \\
\hline Ibovespa & 1.00 & 0.42 & 0.36 & 0.18 \\
\hline Micex & 0.42 & 1.00 & 0.41 & 0.16 \\
\hline Sensex & 0.36 & 0.41 & 1.00 & 0.25 \\
\hline Shanghai & 0.18 & 0.15 & 0.24 & 1.00 \\
\hline
\end{tabular}

The index returns of all the four economies were tested for existence of significant correlation. As is visible from Table 4, none of the BRIC economies have shown a fair amount of correlation as far as returns are concerned.

\subsection{Shannon Entropy}

\begin{tabular}{|c|c|c|c|c|}
\hline & \multicolumn{4}{|c|}{ Table 5 : Shannon Entropy and Standard Deviation } \\
\hline Index & $\begin{array}{c}\text { Standard } \\
\text { Deviation(S.D.) }\end{array}$ & Shannon Entropy (S.E.) & Rank S.D. & Rank S.E. \\
\hline Ibovespa & 1.88 & 0.999252 & 2 & 1 \\
\hline Micex & 2.42 & 0.999949 & 4 & 4 \\
\hline Sensex & 1.71 & 0.998981 & 3 & 2 \\
\hline Shanghai & 1.76 & 0.999333 & 4 \\
\hline
\end{tabular}

Shannon Entropy was calculated for the four indices using Equation no. (11). The values of Shannon Entropy were interpreted in terms of volatility. Of the four BRIC economies the Russian market index Micex is found to be most volatile and Indian market index Sensex is the least volatile. The reason could be attributed to short history of the Russian stock market and strong fundamentals of the Indian markets. The Russian stock market show relatively more rapid response to the frequent market changes both in the local as well as global markets. The other reason could be little high liquidity instruments available in the market. On the other hand, the Indian stock market enjoys strong fundamentals, demographic dividend and robust policies adopted by the policy makers. In the events of high volatility, the policy makers take immediate actions to get the markets under control. 
Further, mixed results were obtained for comparison between Shannon Entropy and Standard deviation. The highest as well as the lowest ranks remain same for findings through both the methods. However, a contradiction as is visible in table is found in ranking of Ibovespa and Shanghai index.

\section{Conclusion}

The question of efficient market hypothesis's validity in financial markets has been raised in a wide number of researches. Moreover, empirical evidence of emerging markets being more inefficient than the developed ones has opened opportunities for the portfolio investors to exploit the predictability in these markets. BRIC economies represent the major emerging markets. A substantial percentage of world's GDP growth is also contributed by them. Hence, it is not surprising that these economies have attracted attention of the whole world.

The conventional view assumes and assesses market efficiency as a static concept. However, a recent strand of literature proposed that market efficiency is an ever evolving time dependent concept. Also, as efficiency tends to change over time, researchers argue that these markets are becoming more efficient with time [9]. This led to a systematic investigation into the underlying dynamics of the major stock exchange indices of these economies. The limitations posed on many widely used forecasting tools due to the impact of non-linear dynamics in stock markets can be overcome by replacing or complementing those tools with the tools of Econophysics. One such tool is Hurst Exponent analysis. The numerical estimate of Hurst is able to quantify the predictability in a fractal time-series. Investigation of the major indices of the economies with respect to Hurst led to interesting results. By plotting it with the indices' closing prices, it was found that Hurst values were able to provide adequate signals in case of Micex, Shanghai and Sensex indices. Also, Chinese market indexShanghai was found to be the most predictable making the market the most inefficient amongst the four economies, whereas, the Brazilian market index- Ibovespa was the most efficient. As inefficiency was found in these markets, another tool of Econophysics was used to measure volatility in these markets. Shannon entropy provides advantage over other statistical techniques as it is not limited by any theoretical or distribution constraints. It was found that the Russian market was the most volatile and the Indian market the least. The findings by standard deviation technique, a popular measure of volatility, also came out to be same for the Russian and Indian market index.

The study has wide implications ranging from investment decisions to policy making. The finding that the Sensex market has been the least volatile amongst the BRIC indices over the period being studied would facilitate those investors (FIIs) who have low risk appetite. Further, the rolling Hurst values plotted along with closing prices in inefficient markets would facilitate investors on deciding on their exit and entry into the market and also, to regulators on when to intervene. The limitations of the conventional techniques overcome by the Statistical Physics tool (Hurst Exponent) and Information theory tool (Shannon Entropy) coupled with neural networks can lead to better forecasting by the users. There can be increased chances of earning abnormal profits by investors and the appropriate policy actions by policy makers will ultimately lead to a more efficient market.

\section{References}

[1] E. Fama, Efficient capital market: a review of theory and empirical work, Journal of Finance, 25, $1970,383-417$.

[2] José A.O. Matos, Sílvio M.A. Gama, Heather J. Ruskin, Adel Al Sharkasi and Martin Crane, Time and scale Hurst exponent analysis for financial markets, 2007.

[3] C.E. Shannon, A mathematical theory of communication, The Bell Technical Journal, 27(4), 1948, 379-423.

[4] E. Peters, Chaos and Order in the Capital Markets: A new view of cycles, prices, and market volatility, 1991, New York: Wiley.

[5] L. Glenn, On Randomness and the NASDAQ Composite, 2007, Available at SSRN 1124991

[6] M. Karamchandani and S. Jain, Indian forex market: Revisited through Hurst exponent and Technical analysis. International Journal of Commerce, Business and Management (IJCBM), Vol. 2, No.4, 2013.

[7] M. Corazza, \& A. G. Malliaris, Multifractality in foreign currency markets, Multinational Finance Journal, 6, 2003, 387-401.

[8] D. Grech and Z. Mazur, Can One Make Any Crash Prediction in Finance Using the Local Hurst Exponent Idea? Physica A, 336, 2003, 133.

[9] D. O. Cajueiro, \& B. M. Tabak, The Hurst exponent over time: testing the assertion that emerging markets are becoming more efficient, Physica A: Statistical Mechanics and its Applications, 336(3), 2004, 521-537.

[10] S. Da Silva, R. Matsushita, I. Gleria, \& A. Figueiredo, Hurst exponents, power laws, and efficiency in the Brazilian foreign exchange market, Economics Bulletin, 7(1), 2007, 1-11.

[11] Jose Alvarez-Ramirez, Jesus Alvarez, Eduardo Rodriguez, and Guillermo Fernandez-Anaya, Time-varying Hurst exponent for US stock markets, Physica A, 387, 2008, 6159-6169.

[12] Cheoljun Eom, Sunghoon Choi, Gabjin Oh and Woo-Sung Jung, Hurst exponent and prediction based on weak-form efficient market hypothesis of stock markets, Physica A, 387, 2008, 4630-4636.

[13] Massimo Guidolin, Stuart Hyde, David Mcmillan and Sadayuki Ono, Non-linear predictability in stock and bond returns: when and where is it exploitable? Research division, Federal Reserve Bank of St. Louis, 2008.

[14] Sonia R. Bentes, Rui Menezes and Diana A. Mendes, Long Memory and Volatility Clustering: is the empirical evidence consistent across stock markets? N.d., 2008.

[15] V. Sakalauskas, \& D. Kriksciuniene, Tracing of stock market long term trend by information efficiency measures, Neurocomputing, 2012. 
[16] M. Mehta, Foreign Exchange Markets, in A. N. Refenes [Ed.] (Neural Networks in the Capital Markets, John Wiley, Chichester,1995) 176-198.

[17] Harold Edwin Hurst, Robert Pearson Black, and Y. M. Simaika, Long-term storage: an experimental study, Constable, 1965.

[18] Noah, Joseph, and operational hydrology, Water resources research, 4(5), 1968, 909-918.

[19] B. B. Mandelbrot, \& J. R. Wallis, Computer Experiments with Fractional Gaussian Noises: Part 3, Mathematical Appendix, Water Resources Research, 5(1), 1969a, 260-267.

[20] B. B. Mandelbrot, \& J. R. Wallis, Robustness of the rescaled range R/S in the measurement of non cyclic long run statistical dependence, Water Resources Research, 5(5), 1969b, 967-988.

[21] B. B. Mandelbrot, A fast fractional Gaussian noise generator, Water Resources Research, 7(3), 1971, 543-553.

[22] S. S. S. Kumar, Square Root of Time Rule and the Indian Stock Market, South Asian Journal of Management, 13(4), 2006.

[23] D. O. Cajueiro, \& B. M. Tabak, Testing for predictability in equity returns for European transition markets, Economic Systems, 30, 2006, 56-78.

[24] X. Z. Tang, E. R Tracy, A. D. Boozer, \& R. Brown, Symbol sequence statistics in noisy chaotic signal reconstruction, Physical Review E, 51(5), 1995, 3871.

[25] X. Z. Tang, E. R Tracy, \& R. Brown, Symbol statistics and spatio-temporal systems, Physica D: Nonlinear Phenomena, 102(3), 1997, 253-261.

[26] M. Lehrman, A. B. Rechester, \& R. B. White, Symbolic analysis of chaotic signals and turbulent fluctuations, In APS Meeting Abstracts (Vol. 1), 1997.

[27] X. Z. Tang \& E. R Tracy, Data compression and information retrieval via symbolization, Chaos: An Interdisciplinary Journal of Nonlinear Science, 8(3), 1998, 688-696.

[28] W. A. Risso, Measuring the informational efficiency in the stock market and its economic effects. Ph.D. Thesis, Department of economics: University of Siena, 2008.

[29] Stock screener. Google Finance. Retrieved September 13, 2013 from http://www.google.com/finance\#stockscreener.

[30] T. T. L. Chong, S. H. S. Cheng, \& E. N. Y. Wong, A Comparison of Stock Market Efficiency of the BRIC Countries, Technology and Investment, 1(4), 2010, 235-238. 